\title{
LOCAL RINGS OF RELATIVELY SMALL TYPE ARE COHEN-MACAULAY
}

\author{
TAKESI KAWASAKI
}

(Communicated by Wolmer V. Vasconcelos)

\begin{abstract}
Let $A$ be a local ring of type $n$. It is known that if $n=1$, then $A$ is Cohen-Macaulay and that if $n=2$ and $A$ is unmixed, then $A$ is CohenMacaulay. Then let $n \geq 3$. What makes $A$ Cohen-Macaulay? We show that if $A$ contains a field and $\hat{A}$ satisfies $\left(S_{n-1}\right)$, then $A$ is Cohen-Macaulay.
\end{abstract}

\section{INTRODUCTION}

Let $A$ be a Noetherian local ring with maximal ideal $\mathfrak{m}$ of dimension $d$. The type of $A$, denoted by $r_{A}(A)$, is defined to be the length of $\operatorname{Ext}_{A}^{d}(A / \mathfrak{m}, A)$. It is well known that if $A$ is a Cohen-Macaulay ring, then the index of reducibility of any parameter ideal for $A$ is equal to the type of $A$, and that Gorenstein rings are characterized as Cohen-Macaulay rings of type one [2]. Vasconcelos conjectured that rings of type one are Gorenstein [17]. This conjecture was first proven by Foxby for rings containing a field and for unmixed local rings [6]. Foxby used the existence of a big-Cohen-Macaulay module and the theory of dualizing complexes. But Roberts proved this conjecture in general [13], using somewhat more elementary methods than those used by Foxby.

Modifying Roberts's argument, Costa, Huneke, and Miller showed that complete local domains of type two are Cohen-Macaulay [4]. Furthermore Marley improved their result and proved that unmixed local rings of type two are Cohen-Macaulay [9].

Concerning these results, the following questions may be raised:

Let $A$ be a Noetherian local ring whose type is known. What makes $A$ CohenMacaulay?

For example, Marley asked if complete local rings of type $n$ which satisfy Serre's condition $\left(S_{n-1}\right)$ are Cohen-Macaulay [9]. We answer Marley's question affirmatively, when $A$ contains a field and $n \geq 3$, and give a generalization for modules.

Received by the editors March 2, 1993.

1991 Mathematics Subject Classification. Primary 13C14, $13 \mathrm{H} 10$.

Key words and phrases. Cohen-Macaulay ring, Cohen-Macaulay module, type of local ring.

The author is partially supported by Grant-Aid for Co-operative Research.

(C) 1994 American Mathematical Society $0002-9939 / 94 \$ 1.00+\$ .25$ per page 


\section{Preliminaries}

In this section, we state some definitions and recall some facts on Serre's condition and on a module with finite local cohomologies. Throughout this paper, $A$ denotes a Noetherian local ring with maximal ideal $\mathfrak{m}$. Let $M$ be a finitely generated $A$-module. We put $\operatorname{Assh}_{A} M=\left\{\mathfrak{p} \in \operatorname{Ass}_{A} M \mid \operatorname{dim} A / \mathfrak{p}=\right.$ $\operatorname{dim} M\}$. We say that $M$ is unmixed (resp. quasi-unmixed) if Ass $_{\hat{A}} \hat{M}=$ $\operatorname{Assh}_{\hat{A}} \hat{M}\left(\right.$ resp. $\left.\operatorname{Min}_{\hat{A}} \hat{M}=\operatorname{Assh}_{\hat{A}} \hat{M}\right)$, where $\hat{A}$ (resp. $\left.\hat{M}\right)$ denotes the $\mathfrak{m}$ adic completion of $A$ (resp. $M$ ) [11]. If $A$ is a homomorphic image of a Cohen-Macaulay ring, then $M$ is unmixed (resp. quasi-unmixed) if and only if $\operatorname{Ass}_{A} M=\operatorname{Assh}_{A} M$ (resp. $\operatorname{Min}_{A} M=\operatorname{Assh}_{A} M$ ) [10, Theorem 31.6].

Definition (2.1). Let $M$ be a finitely generated $A$-module of dimension $s$. For $i \geq 0$, the $i$-th Bass number of $M$, denoted by $\mu_{A}^{i}(M)$, is defined to be the length of $\operatorname{Ext}_{A}^{i}(A / \mathfrak{m}, M)$. Let $I^{\bullet}$ be the minimal injective resolution of $M$ and $E$ be the injective envelope of the residue field of $A$. Then the $i$-th Bass number is equal to the number of copies of $E$ which appear in $I^{i}$ as direct summands. We refer the reader to [2] and $[10, \S 18]$ for details. In particular, the type of $M$, denoted by $r_{A}(M)$, is defined to be the $s$-th Bass number of $M$.

Definition (2.2) (Serre's condition). Let $t$ be an integer. A finitely generated $A$-module $M$ is said to satisfy $\left(S_{t}\right)$ if $\operatorname{depth} M_{\mathfrak{p}} \geq \min \left\{t, \operatorname{dim} M_{\mathfrak{p}}\right\}$ for all $\mathfrak{p}$ in $\operatorname{Supp} M$.

Suppose that $A$ is a homomorphic image of a Cohen-Macaulay ring. Then $M$ satisfies $\left(S_{t}\right)$ if and only if $\hat{M}$ satisfies it [10, §23].

Now we state the definition of a module with finite local cohomologies or a generalized Cohen-Macaulay module, and we give characterizations of it. We refer the reader to [7], [14], or [16] for details.

Definition (2.3). A finitely generated $A$-module $M$ is referred to as a module with finite local cohomologies if $H_{\mathfrak{m}}^{i}(M)$ has finite length for all $i \neq \operatorname{dim} M$, where $H_{\mathfrak{m}}^{i}(-)$ denotes the $i$-th local cohomology functor with respect to $\mathfrak{m}$.

It is obvious that $M$ is a module with finite local cohomologies if and only if $\hat{M}$ is also.

Lemma (2.4) [7, Theorem 37.4]. Let $M$ be a finitely generated A-modüle.

(1) If $M$ is a module with finite local cohomologies, then $\operatorname{Min}_{A} M=$ Assh $_{A} M$ and $M_{\mathfrak{p}}$ is a Cohen-Macaulay $A_{\mathfrak{p}}$-module for all $\mathfrak{p}$ in Supp $M \backslash\{\mathfrak{m}\}$.

(2) If $A$ is a homomorphic image of a Cohen-Macaulay ring, then the converse to (1) holds.

Lemma (2.5) [7, Theorem 37.10]. Let $M$ be a finitely generated A-module of dimension $d$. Then the following statements are equivalent:

(1) $M$ is a module with finite local cohomologies;

(2) $\sup \left\{\ell_{A}(M / \mathbf{x} M)-e_{\mathbf{x A}}(M) \mid \mathbf{x}=x_{1}, \ldots, x_{d}\right.$ is a system of parameters for $M\}$ is finite, 
where $\ell_{A}(N)$ denotes the length of an $A$-module $N$ and $e_{\mathbf{x A}}(M)$ denotes the multiplicity of $M$ with respect to $\mathbf{x} A$. If $M$ satisfies these equivalent statements, then this supremum is equal to $\sum_{i=0}^{d-1}\left(\begin{array}{c}d-1 \\ i\end{array}\right) \ell_{A}\left(H_{\mathfrak{m}}^{i}(M)\right)$. Furthermore $\ell_{A}(M / \mathbf{x} M)-e_{\mathbf{x} A}(M)$ is equal to the supremum if and only if

$$
\mathbf{x} H_{\mathfrak{m}}^{i}\left(M /\left(x_{1}, \ldots, x_{j}\right) M\right)=0 \text { for all } i+j<d .
$$

The system of parameters which satisfies these equivalent conditions is called a standard system.

\section{MAIN THEOREM AND ITS PROOF}

This section is devoted to a proof of the following theorem.

Theorem (3.1). Let $A$ be a local ring containing a field and suppose that $A$ is a homomorphic image of a Cohen-Macaulay ring. Let $n$ be a positive integer.

(i) If $A$ satisfies the following conditions:

(1) $r_{A}(A) \leq n$

(2) A satisfies $\left(S_{n-1}\right)$;

(3) $\operatorname{Min}_{A} A=\operatorname{Assh}_{A} A$,

then $A$ is Cohen-Macaulay.

(ii) Let $M$ be a finitely generated A-module. If $M$ satisfies the following conditions:

(1) $r_{A}(M) \leq n$;

(2) $M$ satisfies $\left(S_{n-1}\right)$;

(3) $\operatorname{Min}_{A} M=\operatorname{Assh}_{A} M$;

(4) $M_{\mathfrak{p}}$ is a Cohen-Macaulay $A_{\mathfrak{p}}$-module for all $\mathfrak{p}$ in $\operatorname{Supp} M$ such that $\operatorname{dim} M_{\mathfrak{p}} \leq n$,

then $M$ is Cohen-Macaulay.

We need the following result to prove this theorem. For any matrix $\varphi, I_{r}(\varphi)$ denotes the ideal generated by the $r$-minors of $\varphi$.

Proposition (3.2). Let $A$ be a local ring containing a field and $\mathfrak{m}$ be the maximal ideal of $A$. We consider a complex

$$
F_{\bullet}: 0 \longrightarrow F_{p} \stackrel{\varphi_{p}}{\longrightarrow} F_{p-1} \longrightarrow \cdots \longrightarrow F_{1} \stackrel{\varphi_{1}}{\longrightarrow} F_{0},
$$

of finite free A-modules with $F_{p} \neq 0$ and $\varphi_{i}\left(F_{i}\right) \subseteq \mathfrak{m} F_{i-1}$ for $i=1, \ldots, p$. Suppose that there is some integer $q \geq 0$ and that following inequalities hold:

$$
\operatorname{dim} A / I_{r_{i}}\left(\varphi_{i}\right) \leq \operatorname{dim} A-q-i, \quad i=1, \ldots, p,
$$

where $r_{i}=\sum_{j=i}^{p}(-1)^{j-i} \operatorname{rank} F_{j}$. Then $r_{i} \geq q+i$ for $i=1, \ldots, p-1$.

Proof. See [3].

Bruns had proved this proposition using the existence of a big-CohenMacaulay module. And he had evaluated Bass numbers of finitely generated modules over local rings by this proposition. But now we are able to obtain strict evaluations for a module with finite local cohomologies. 
Proposition (3.3). Let $A$ be a local ring containing a field and $\mathfrak{m}$ be the maximal ideal of $A$. Let $M$ be a non-Cohen-Macaulay A-module with finite local cohomologies. If we put $d=\operatorname{dim} A, s=\operatorname{dim} M$, and $t=\operatorname{depth} M$, then we have

(1)

$$
\mu_{A}^{i}(M) \geq \begin{cases}1, & i=t, \\ \mu_{A}^{t}(M)+d-1, & i=t+1, \\ 2(d+t-i)+1, & t+1<i<s .\end{cases}
$$

(2) $r_{A}(M) \geq \min \{t+d-s+2, d\}$.

(3) If $M=A$, then $r_{A}(A) \geq t+2$.

Proof. Without loss of generality, we may assume that $A$ is complete. Let

$$
0 \rightarrow M \rightarrow I^{0} \rightarrow I^{1} \rightarrow \cdots \rightarrow I^{i} \rightarrow \cdots
$$

be a minimal injective resolution of $M$ and apply $H_{\mathfrak{m}}^{0}(-)$ to $I^{\bullet}$. Then we obtain the complex

$$
H_{\mathrm{m}}^{0}\left(I^{\bullet}\right): 0 \rightarrow E^{\mu_{A}^{t}(M)} \rightarrow E^{\mu_{A}^{t+1}(M)} \rightarrow \cdots \rightarrow E^{\mu_{A}^{i}(M)} \rightarrow E^{\mu_{A}^{i+1}(M)} \rightarrow \cdots
$$

where $E$ is the injective envelope of the residue field of $A$. We note that local cohomology modules of $M$ are cohomology modules of this complex. We consider the following complex

$$
F_{\bullet}=\operatorname{Hom}_{A}\left(H_{\mathfrak{m}}^{0}\left(I^{\bullet}\right), E\right): \cdots \rightarrow F_{i+1} \stackrel{\varphi_{i}}{\rightarrow} F_{i} \rightarrow \cdots \rightarrow F_{t+1} \stackrel{\varphi_{i}}{\rightarrow} F_{t} \rightarrow 0 .
$$

Since the endomorphism ring of $E$ is isomorphic to $A, F_{i}$ is an $A$-free module of rank $\mu_{A}^{i}(M)$. Furthermore $H_{i}\left(F_{\bullet}\right)=\operatorname{Hom}_{A}\left(H_{\mathfrak{m}}^{i}(M), E\right)$ has finite length for $i \neq s$, and $\operatorname{Supp} H_{s}\left(F_{0}\right)$ is equal to $\operatorname{Supp} M$, because $H_{s}\left(F_{0}\right)$ is the canonical module of $M$ [15]. So we put $r_{t}=\mu_{A}^{t}(M)$ and $r_{i}=\mu_{A}^{i}(M)-r_{i-1}$ for $i>t$. Then $I_{r_{i}}\left(\varphi_{i}\right)$ is an m-primary ideal for all $i<s$ and $r_{s-1}<\mu_{A}^{s}(M)$. If $s>t+1$, then we obtain $r_{i} \geq d+t-i$ for $t+1 \leq i<s$, in applying Proposition (3.2) to the complex $\operatorname{Hom}_{A}\left(F_{0}, A\right)$, let $p=s-t$ and $q=d-s+t$. On the other hand, if $s=t+1$, then we have $\mu_{A}^{s}(M)-\mu_{A}^{s-1}(M)+1 \geq d$, by using Eagon-Northcott's theorem [5]. Now (1) and (2) are proved.

If $M=A$ and $r_{A}(A)<t+2$, then $t$ must be equal to $d-1, \mu_{A}^{d}(A)=d$, $\mu_{A}^{d-1}(A)=1$ and $\varphi_{d-1}=\left(x_{1}, \cdots, x_{d}\right)$, where $\mathbf{x}=x_{1}, \ldots, x_{d}$ is a system of parameters for $A$. Moreover $\mathbf{x}$ is a standard system by Lemma (2.5), hence $\ell_{A}(A / \mathbf{x} A)-e_{\mathbf{x} A}(A)=\ell_{A}\left(\operatorname{Hom}_{A}(A / \mathbf{x} A, E)\right)$. This implies that $e_{\mathbf{x} A}(A)=0$, which is a contradiction.

Proof of Theorem (3.1). (i) We work by induction on $d=\operatorname{dim} A$. There is nothing to prove if $d<n$. If $d \geq n$, then $A_{\mathfrak{p}}$ is Cohen-Macaulay for all $\mathfrak{p}$ in Spec $A \backslash\{\mathfrak{m}\}$ because $r_{A_{\mathfrak{p}}}\left(A_{\mathfrak{p}}\right) \leq r_{A}(A)$ (see [6] or [12]). Hence $A$ is a ring with finite local cohomologies. If $A$ is not Cohen-Macaulay then $n \geq r_{A}(A) \geq$ depth $A+2$. This contradicts the fact that $\operatorname{depth} A \geq n-1$.

(ii) is proved in the same way.

Finally we answer Marley's question.

Corollary (3.4). Let $A$ be a Noetherian local ring containing a field. If $r_{A}(A)=$ $n \geq 3$ and $\hat{A}$ satisfies $\left(S_{n-1}\right)$, then $A$ is Cohen-Macaulay. 
Proof. If $\hat{A}$ satisfies $\left(S_{2}\right)$, then $\operatorname{Ass}_{\hat{A}} \hat{A}=\operatorname{Assh}_{\hat{A}} \hat{A}$ [1]. Hence we can apply Theorem (3.1) to $\hat{A}$.

\section{EXAMPLES}

In this section, we give some examples and conjectures.

Example (4.1). Let $k$ be a field and

$$
A=k \llbracket x, y, z \rrbracket /(x y, x z)=k \llbracket x, y, z \rrbracket /(x) \cap(y, z),
$$

where $x, y, z$ are indeterminates over $k$. Then $\operatorname{dim} A=2, r_{A}(A)=2$ and $A$ satisfies $\left(S_{1}\right)$ (see [4]). But $A$ is not Cohen-Macaulay, because $A$ is not quasi-unmixed.

Rings of type one are automatically quasi-unmixed, because they are CohenMacaulay. But local rings of type two are not necessarily unmixed. Hence we cannot omit condition (3) of Theorem (3.1).

Example (4.2). Let $k$ be a field and $A$ be a formal power series ring $k \llbracket x_{1}, \ldots, x_{d} \rrbracket$ where $d>1$. $\left(K_{\bullet}, \varphi_{\bullet}\right)$ denotes the Koszul complex generated by $x_{1}, \ldots, x_{d}$ over $A$. Then $\operatorname{Im} \varphi_{d-1}$ is a non-Cohen-Macaulay module with finite local cohomologies. Because, by considering the following exact sequence:

$$
0 \rightarrow \operatorname{Im} \varphi_{1} \rightarrow K_{0} \rightarrow k \rightarrow 0,
$$

we have

$$
\mu_{A}^{i}\left(\operatorname{Im} \varphi_{1}\right)= \begin{cases}\left(\begin{array}{c}
d \\
i-1
\end{array}\right), & 1 \leq i \leq d, \\
0, & \text { otherwise }\end{cases}
$$

and

$$
H_{\mathfrak{m}}^{i}\left(\operatorname{Im} \varphi_{1}\right)= \begin{cases}H_{\mathfrak{m}}^{d}(A), & i=d, \\ A / \mathfrak{m}, & i=1, \\ 0, & \text { otherwise. }\end{cases}
$$

In the same way, we obtain

$$
\mu_{A}^{i}\left(\operatorname{Im} \varphi_{d-1}\right)= \begin{cases}d, & i=d \\ 1, & i=d-1 \\ 0, & \text { otherwise }\end{cases}
$$

and

$$
H_{\mathfrak{m}}^{i}\left(\operatorname{Im} \varphi_{d-1}\right)= \begin{cases}A / \mathfrak{m}, & i=d-1, \\ 0, & i \neq d, d-1 .\end{cases}
$$

Thus $\operatorname{Im} \varphi_{d-1}$ is a module with finite local cohomologies such that its type is equal to its dimension.

This example means that Theorem (3.3) is best possible for modules, and that we cannot omit condition (4) of Theorem (3.1). Is Proposition (3.3) best possible for rings? Let $k$ be a field and $A=k \llbracket x, y \rrbracket /\left(x^{2}, x y\right)$. Then $A$ is a ring with finite local cohomologies and $r_{A}(A)=\operatorname{depth} A+2$ [4]. Hence Proposition (3.3) is also the best possible for rings, but the depth of $A$ is zero. Does there exist such a domain? We have a non-Cohen-Macaulay domain $A$ with finite local cohomologies such that $r_{A}(A)=\operatorname{depth} A+3$. 
Example (4.3). Let $k$ be a field and $A=k \llbracket s^{2}, s^{3}, s t, t \rrbracket$. Then $A$ is a ring with finite local cohomologies of dimension two, and depth one. The type of $A$ is equal to four because $A$ is isomorphic to

$$
k \llbracket a, b, c, d \rrbracket /\left(a^{3}-b^{2}, c^{2}-a d^{2}, a c-b d, b c-a^{2} d\right) .
$$

So if we put $B=A / t A$, then $B$ is isomorphic to

$$
k \llbracket a, b, c \rrbracket /\left(a^{3}-b^{2}, c^{2}, a c, b c\right) .
$$

The resolution of $k$ over $B$ begins

$$
0 \longleftarrow k \longleftarrow B \stackrel{\phi_{1}}{\longleftarrow} B^{3} \stackrel{\phi_{2}}{\longleftarrow} B^{7}
$$

where $\phi_{1}=(a b c)$ and

$$
\phi_{2}=\left(\begin{array}{ccccccc}
c & 0 & 0 & 0 & 0 & a^{2} & b \\
0 & c & 0 & 0 & 0 & -b & -a \\
0 & 0 & c & a & b & 0 & 0
\end{array}\right) .
$$

Thus $r_{A}(A)=r_{B}(B)=\ell_{B}\left(\operatorname{Ext}_{B}^{1}(k, B)\right)$, which is the length of the homology of the complex

$$
B^{7} \stackrel{{ }^{t} \phi_{2}}{\longleftarrow} B^{3} \stackrel{{ }^{t} \phi_{1}}{\longleftarrow} B .
$$

This is minimally generated by classes of

$$
\left(\begin{array}{l}
c \\
0 \\
0
\end{array}\right),\left(\begin{array}{l}
0 \\
c \\
0
\end{array}\right),\left(\begin{array}{l}
0 \\
0 \\
c
\end{array}\right),\left(\begin{array}{c}
b \\
-a^{2} \\
0
\end{array}\right) .
$$

Question (4.4). Does there exist a complete local domain $A$ that is not CohenMacaulay but that has $r_{A}(A)=3$ ?

We can prove a similar result on "classical type" (that is, the supremum of the index of reducibility of parameter ideals) [8]. This leads one to make the following conjectures.

Conjecture (4.5). Let $A$ be a complete unmixed local ring of type $n$. If $A_{\mathfrak{p}}$ is Cohen-Macaulay for all $\mathfrak{p}$ in $\operatorname{Spec} A$ such that $\mathrm{ht} \mathfrak{p}<n$, then $A$ is CohenMacaulay.

Conjecture (4.6). Let $A$ be a complete local ring and $M$ be a finitely generated unmixed A-module of type $n$. If $M_{\mathfrak{p}}$ is a Cohen-Macaulay $A_{\mathfrak{p}}$-module for all $\mathfrak{p}$ in $\operatorname{Supp} M$ such that $\operatorname{dim} M_{\mathfrak{p}} \leq n$, then $M$ is Cohen-Macaulay.

\section{ACKNOWLEDGMENT}

The author is grateful to Professor S. Goto for his hearty guidance during this research.

\section{REFERENCES}

1. Y. Aoyama and S. Goto, On the endomorphism ring of the canonical module, J. Math. Kyoto Univ. 25 (1985), 21-30.

2. H. Bass, On the ubiquity of Gorenstein rings, Math. Z. 82 (1963), 8-28.

3. W. Bruns, The Evans-Griffith syzygy theorem and Bass numbers, Proc. Amer. Math. Soc. 115 (1992), 939-946. 
4. D. Costa, C. Huneke, and M. Miller, Complete local domains of type two are CohenMacaulay, Bull. London Math. Soc. 17 (1985), 29-31.

5. J. A. Eagon and D. G. Northcott, Ideals defined by matrices and a certain complex associated with them, Proc. Roy. Soc. London Ser. A 296 (1962), 188-204.

6. H.-B. Foxby, On the $\mu^{i}$ in a minimal injective resolution II, Math. Scand. 41 (1977), 19-44.

7. M. Herrmann, S. Ikeda, and U. Orbanz, Equimultipilicity and Blowing up, Springer-Verlag, Berlin, Heidelberg, and New York, 1988.

8. T. Kawasaki, On the index of reducibility of parameter ideal and Cohen-Macaulayness in a local ring, J. Math. Kyoto Univ. 34 (1994), 219-226.

9. T. Marley, Unmixed local rings of type two are Cohen-Macaulay, Bull. London Math. Soc. 23 (1991), 43-45.

10. H. Matsumura, Commutative ring theory, Cambridge Univ. Press, New York, 1986.

11. M. Nagata, Local rings, Interscience Tracts Pure Appl. Math., vol. 13, Interscience, New York and London, 1962.

12. P. Roberts, Homological invariants of modules over commutative rings, Sém. Math. Sup., Presses Univ. Montréal, Montréal, 1980.

13. __ Rings of type 1 are Gorenstein, Bull. London Math. Soc. 15 (1983), 48-50.

14. P. Schenzel, N. V. Trung, and N. T. Cuong, Verallgemeinerte Cohen-Macaulay-Moduln, Math. Nachr. 85 (1978), 57-73.

15. J. Stückrad and W. Vogel, Buchsbaum rings and applications, Springer-Verlag, Berlin, Heidelberg, and New York, 1986.

16. N. V. Trung, Toward theory of generalized Cohen-Macaulay modules, Nagoya Math. J. 102 (1986), 1-49.

17. W. V. Vasconcelos, Divisor theory in module categories, North-Holland Math. Stud., vol. 14, North-Holland, Amsterdam, 1975.

Deprtment of Mathematics, Tokyo Metropolitan University, Minami-Ohsawa 1-1, HACHIOJI-SHI, TOKYO 192-03, JAPAN

E-mail address: kawasaki@math.metro-u.ac.jp 